in vivo $32: 1609-1615$ (2018)

doi:10.21873/invivo.11421

\title{
Calcium Phosphate Cement Paste Injection as a Fiducial Marker of Cervical Cancer
}

\author{
ICHIRO OGINO ${ }^{1}$, MASAKAZU KITAGAWA ${ }^{2}$, SHIGENOBU WATANABE ${ }^{1}$, \\ HIROSHI YOSHIDA ${ }^{3}$ and MASAHARU HATA ${ }^{4}$ \\ ${ }^{1}$ Department of Radiation Oncology, and ${ }^{2}$ Department of Gynecology, \\ Yokohama City University Medical Center, Yokohama, Japan; \\ ${ }^{3}$ Center of Gynecologic Endoscopy and Surgery, Yokohama Municipal Citizen's Hospital, Yokohama, Japan; \\ ${ }^{4}$ Division of Radiation Oncology, Department of Oncology, \\ Yokohama City University Graduate School of Medicine, Yokohama, Japan
}

\begin{abstract}
Background/Aim: Calcium phosphate cement $(C P C)$ is used to fill bone voids in dental, orthopedic, and craniofacial applications. This study evaluated CPC marker as an injectable non-metallic fiducial marker. Materials and Methods: Six patients received 3-5 injections of CPC paste placed at a depth of $10 \mathrm{~mm}$ into tumors of the cervix before treatment planning CT (TPCT). Patients were treated with external-beam radiotherapy (EBRT) and high-dose rate brachytherapy $(B T)$. We investigated marker visibility on cone-beam CT (CBCT), T2-weighted MRI, and interfraction of the marker motion for cervical cancer patients. Results. Of a total of 22 visible CPC markers at TPCT, 17 CPC markers were visible on the first $C B C T$. Excluding one patient, all markers were visible on $C B C T$ during EBRT. Of 16 visible $C P C$ markers on $C B C T, 13 C P C$ markers were visible on the magnetic resonance imaging (MRI) obtained before BT. For CPC marker centroid movement, the meanof-means/systematic variation/random variation were $0.2 / 0.4 / 1.4,-1.6 / 5.1 / 4.1$, and $-3.4 / 2.1 / 2.8 \mathrm{~mm}$ for the leftright, dorsal-ventral, and cranial-caudal directions, respectively. Conclusion: This is the first report of a CPC marker injected into tumors of the cervix. It can be visualized on CBCT and MRI with reductions in marker loss and artifacts.
\end{abstract}

This article is freely accessible online.

Correspondence to: Ichiro Ogino, MD, Department of Radiation Oncology, Yokohama City University Medical Center, 4-57 Urahune-cho, Minami-ku, Yokohama, Kanagawa Prefecture, Japan. Tel: +81 452615656, e-mail: ogino1ro@urahp.yokohama-cu.ac.jp

Key Words: Calcium phosphate cement, injectable fiducial marker, cone beam computed tomography, magnetic resonance imaging, cervical cancer.
As experience with image-guided radiotherapy (IGRT) grows, several studies using small gold markers (GM) for different disease sites have demonstrated increased accuracy in the delineation of the target and for patient setup $(1,2)$.

Cone-beam computed tomography (CBCT) is an accurate and precise tool increasingly used for IGRT. This technology is composed of a kilovoltage $\mathrm{X}$-ray $(\mathrm{kV})$ source mounted orthogonally to the megavoltage treatment beam to acquire 3-dimensional (3D) volumetric images. Because of the poor soft-tissue contrast of CBCT, fiducial markers have been used as surrogates of the tumor position. Higher target accuracy matching has been reported using CBCT with implanted GMs than CBCT with soft tissue marking or 2D orthogonal images with implanted GMs (1). Although CBCT provides superior verification of the tumor position with implanted GMs, the higher density of GMs generates larger artifacts on CT images and compromises image quality and interpretation during on-line target localization and verification.

Calcium phosphate cement (CPC) is widely used as a bone substitute (3-5). We injected CPC paste as a fiducial marker in this study. The aim of this study was to evaluate a CPC marker as an injectable nonmetallic fiducial marker. Before starting 3D-based brachytherapy (BT) for cervical cancer in our institute, we investigated CPC marker visibility on CBCT and T2-weighted magnetic resonance imaging (MRI) for cervical cancer patients.

\section{Materials and Methods}

Patients and treatment. A prospective study was performed, including 6 cervical cancer patients diagnosed between July 2014 and January 2016. Patients were treated with external-beam radiotherapy (EBRT) to the whole pelvis with a total prescribed dose of 48.6-50.4 Gy delivered in 27-28 fractions. After 5 EBRT fractions/week were delivered at 34.2-45 Gy, two to three sessions of fractionated high-dose rate intracavitary BT were performed. The 


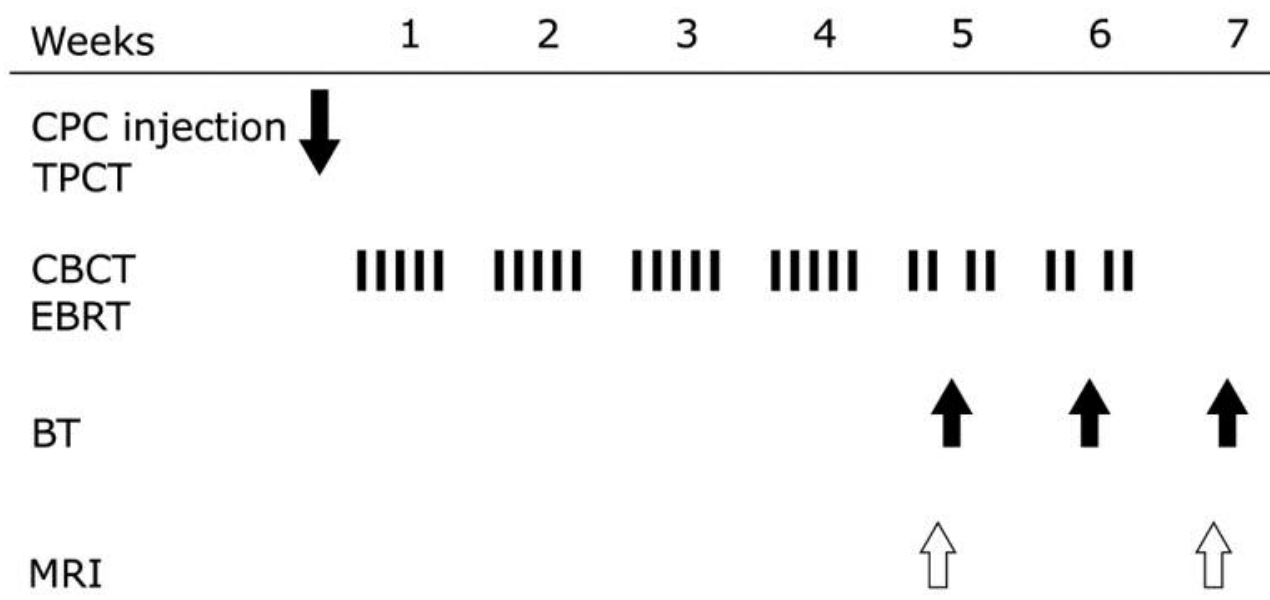

Figure 1. Time schedules of CPC paste injection, TPCT, CBCT, EBRT, and MRI. Patients with 3 sessions of BT.

dose per fraction planned at Point A was 5-6 Gy/week. The treatment schedule included a program of 4 EBRT fractions/week combined with 1 session of BT per week. The final BT was performed within one week after the completion of EBRT. All except one patient received concurrent cisplatin at $40 \mathrm{mg} / \mathrm{m}^{2}$ weekly. This study was approved by the Institutional Committee (approval number: D1603029). Written informed consent for study participation was obtained from all patients.

CPC paste injections and obtained images. Biopex-R (Mitsubishi Materials Corporation, Tokyo, Japan), the injectable CPC paste, was prepared by manual mixing of $1 \mathrm{~g}$ of powder and $0.5 \mathrm{ml}$ of liquid components for $1 \mathrm{~min}$. With the patient in the lithotomy position, $0.1-0.2 \mathrm{ml}$ of the paste was injected using a disposable syringe with a 19-gauge long needle using a speculum for visualization. Six patients received 3-5 injections of CPC paste placed at a depth of $10 \mathrm{~mm}$ into tumors of the cervix.

Treatment planning CT (TPCT) was obtained $2 \mathrm{~h}$ after the injection using a four-slice CT scanner (Lightspeed; GE Medical System, Waukesha, WI, USA) with custom immobilization and a $2.5-\mathrm{mm}$ slice thickness. The TPCT slice dimension was $512 \times 512$ pixels with a $1 \times 1-\mathrm{mm}^{2}$ pixel size.

EBRT was initiated 2 days after the injection. In each fraction, the CBCT scan was acquired before dose delivery. CBCT images were acquired by an on-board CBCT system (Synergy; Elekta AB, Stockholm, Sweden) with settings of $120 \mathrm{kV}, 25 \mathrm{~mA}$, and $40 \mathrm{~ms}$ per frame. The image detector was shifted off-axis by $11.5 \mathrm{~cm}$ in the lateral direction. After the couch position was verified, CBCTs were acquired using the Elekta preset for the pelvis with 650 projections over an angle of $360^{\circ}$. The reconstructed CBCT scans consisted of $410 \times 410 \times 264$ voxels (collimated field of view $(\mathrm{FOV})$ ), with voxel dimensions of $1.0 \times 1.0 \times 1.0 \mathrm{~mm}^{3}$. Patients were positioned with reference to their skin marks, and position verification was based on registration of the bony structures in the CBCT images corresponding to TPCT images.

Visibility of the markers using T2-weighted MRI was also assessed before the first and final BT sessions. A 1.5 Tesla open MRI system (Magnetom Avanto; Siemens, Erlangen, Germany) was used to collect sequential axial images. Fifty axial images were acquired for T2-weighted turbo spin-echo sequences $(\mathrm{TR}=5970 \mathrm{~ms}$, $\mathrm{TE}=102 \mathrm{~ms}$, slice thickness $=3 \mathrm{~mm}, 145^{\circ}$ flip angle, matrix $256 \times 256$, FOV $200 \mathrm{~mm}, 0.8-\mathrm{mm}$ pixels).

Time schedules for obtaining the images are presented in Figure 1.

Analysis. CBCT images for each fraction and two MR images were rigidly registered to the TPCT with respect to the bony anatomy, using Pinnacle ${ }^{3}$ treatment planning system version 9.2 (Philips Radiation Oncology Systems, Milpitas, CA, USA). Alignment of bony anatomies was adjusted automatically using gray-value match after manual registration. Therefore, any setup error was assumed to have been removed. Visibility of the markers using TPCT, CBCT, and T2-weighted MRI was assessed. The markers were determined visible when they were identical by two expert radiation oncologists.

To confirm the visibility of the markers, we investigated interfraction of the marker centroid motion. CPC marker analysis was performed in the $\mathrm{X}$ (left-right), $\mathrm{Y}$ (dorsal-ventral), and $\mathrm{Z}$ (cranial-caudal) coordinate system. To evaluate interfraction of the marker centroid motion, daily CBCT images were compared with the first CBCT image instead of the TPCT image as the CPC marker was shifted by the edematous tumor after the injections. The average position in the repeat CBCT scans, indicated by $m i$ for patient $i$, was calculated as the mean of the marker centroid displacement observed in the treatment fractions. The overall mean deviation, $\mathrm{M}$, was characterized by the average of all $m i$. The random variation $(\sigma)$ was defined as the square root of the average variance. The systematic variation $(\Sigma)$ was defined as the standard deviation of the averages (6).

\section{Results}

The procedure was well-tolerated. There was no interruption of the implantation due to pain or hemorrhage. There were no other procedure-related complications during or after CPC marker injection. 
Table I. Summary of six patients.

\begin{tabular}{|c|c|c|c|c|c|c|c|c|}
\hline $\begin{array}{l}\text { Patient } \\
\text { number }\end{array}$ & $\begin{array}{l}\text { Age } \\
\text { (years) }\end{array}$ & Stage & $\begin{array}{l}\text { Hemoglobin before } \\
\text { CPC injection }(\mathrm{g} / \mathrm{dl})\end{array}$ & $\begin{array}{l}\text { Total EBRT } \\
\text { dose (Gy) }\end{array}$ & $\begin{array}{c}\text { Total BT } \\
\text { dose (Gy) }\end{array}$ & $\begin{array}{c}\text { Concurrent } \\
\text { chemotherapy }\end{array}$ & $\begin{array}{c}\text { Tumor volume } \\
\text { before EBRT* }\left(\mathrm{cm}^{3}\right)\end{array}$ & $\begin{array}{l}\text { Tumor volume of } \\
\text { last MRI** }\left(\mathrm{cm}^{3}\right)\end{array}$ \\
\hline 1 & 87 & $3 b$ & 10.6 & 48.6 & 11.0 & No & 49.4 & 2.7 \\
\hline 2 & 71 & $4 b$ & 12.1 & 50.4 & 12.0 & Yes & 91.7 & 1.1 \\
\hline 3 & 45 & $4 b$ & 11.3 & 50.4 & 18.0 & Yes & 67.2 & 8.4 \\
\hline 4 & 54 & $3 b$ & 12.5 & 50.2 & 17.8 & Yes & 56.7 & 3.9 \\
\hline 5 & 64 & $2 b$ & 9.4 & 50.4 & 17.0 & Yes & 250.7 & 7.5 \\
\hline 6 & 41 & $1 \mathrm{~b} 2$ & 11.7 & 50.4 & 18.0 & Yes & 49.1 & 2.4 \\
\hline
\end{tabular}

CPC: Calcium phosphate cement; EBRT: external-beam radiotherapy; BT: brachytherapy. *Volume measured at staging MRI, **MRI obtained before last BT.

Table II. Number of markers visible in TPCT, CBCT and MRI.

\begin{tabular}{|c|c|c|c|c|c|c|}
\hline Patient number & ТPCT & First CBCT & СBCT before $\mathrm{BT}^{*}$ & First MRI** & Last CBCT & Last MRI $\#$ \\
\hline 1 & 5 & 4 & 4 & 3 & 4 & 3 \\
\hline 2 & 3 & 2 & 2 & 2 & 2 & 2 \\
\hline 3 & 3 & 3 & 3 & 3 & 3 & 3 \\
\hline 4 & 3 & 3 & 3 & 3 & 3 & 3 \\
\hline 5 & 3 & 1 & 0 & 0 & 0 & 0 \\
\hline 6 & 5 & 4 & 4 & 2 & 4 & 2 \\
\hline Total & 22 & 17 & 16 & 13 & 16 & 13 \\
\hline
\end{tabular}

TPCT: Treatment planning CT; CBCT: cone-beam CT; BT: brachytherapy. *CBCT image obtained within 24 h before first MRI, **MRI obtained before first BT, "MRI obtained before last BT.

The clinical data and numbers of CPC markers in each of the images for the 6 patients are shown in Tables I and II. Of the 3 CPC makers visible on TPCT in patient 5, only one marker was visible on the first CBCT, and this marker was not visible after 14 sessions of CBCT. This patient had the largest tumor volume and lowest hemoglobin level by bleeding before CPC marker injection. Of the 22 total visible CPC markers on TPCT, 5 CPC markers were not visible on the first CBCT, before the initial EBRT. Except for patient 5, all invisible CPC markers on the first CBCT were located in the center of the cervix near the cervical os on TPCT. All visible CPC markers on the first CBCT were visible on the last CBCT obtained after one or two sessions of BT.

Of the 16 visible CPC markers on CBCT before the first $\mathrm{BT}$ and the last $\mathrm{CBCT}, 13 \mathrm{CPC}$ markers were visible on the first and last MRI. The signal intensity of CPC was low. All invisible markers on MRI were located adjacent to the cervical canal on CBCT. Figure 2 demonstrates the visible CPC markers in 5 patients.

Patient 2 was excluded from the interfraction study of the marker motion, as the isocenter of the treatment field was located cranially and the half beam block and FOV of CBCT did not include CPC markers in the entire session. Each CPC marker was identified by the size and shape on CBCT during
EBRT. With regard to the first CBCT scan, the mean-ofmeans/systematic variation/random variation of visible CPC markers from the second to 27th fraction of CBCT of 4 patients were $0.2 / 0.4 / 1.4,-1.6 / 5.1 / 4.1$, and $-3.4 / 2.1 / 2.8 \mathrm{~mm}$ for the left-right, dorsal-ventral, and cranial-caudal directions, respectively.

\section{Discussion}

The essential criteria of fiducial markers for IGRT were visibility in the radiologic modalities involved in radiotherapeutic treatment planning and image guidance, the low production of imaging artifacts, and low perturbation of the therapeutic dose to the target volume (7).

GMs were the most widely used fiducial markers. Because the density of GMs is high, they induce markedly bright and dark streak artifacts on TPCT and CBCT images. These streak artifacts produced by GMs make treatment planning extremely difficult by obscuring the contour of soft tissue targets and reducing the accuracy in fiducial-based localization (8). The presence of the GM also affects the dose distribution near the GM surface $(9,10)$. Besides GM, polymeric, nitinol, and carbon-coated markers are new, commercially available solid markers. These new types of 

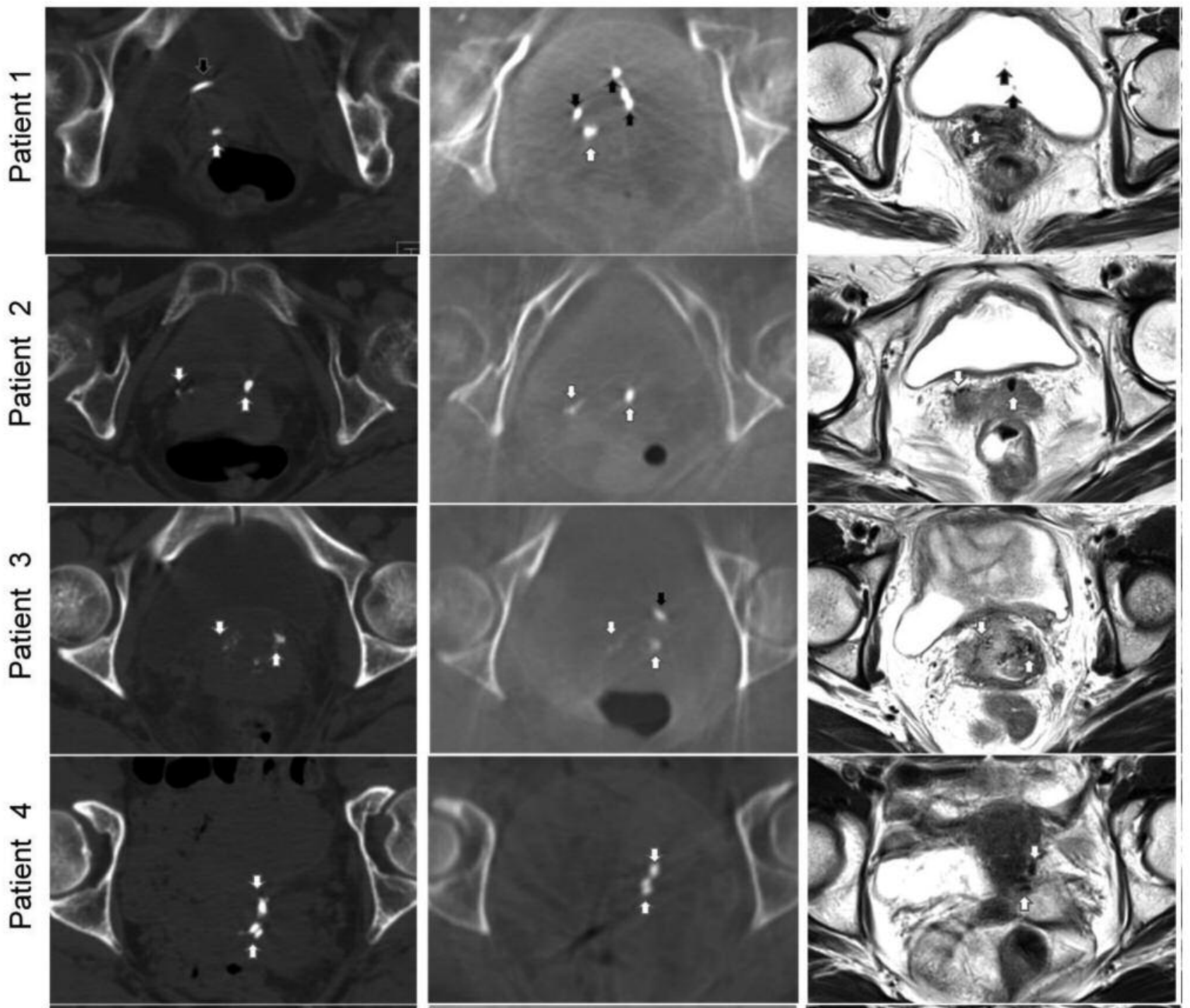

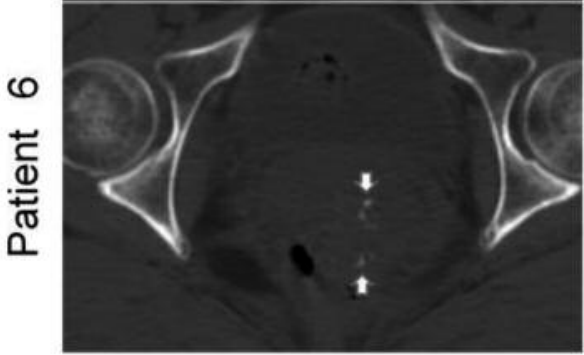

TPCT

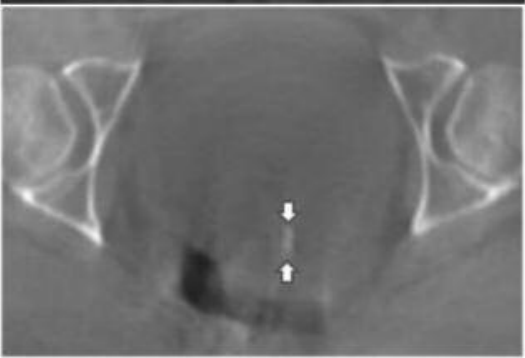

CBCT before first BT

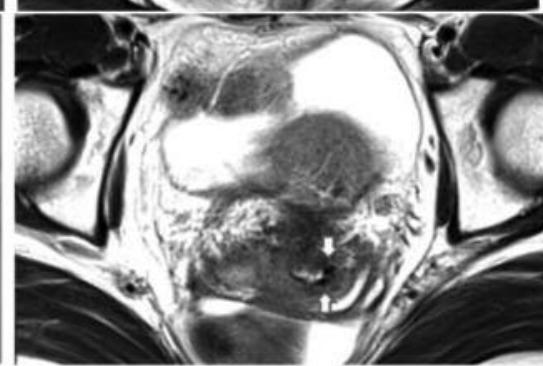

MRI before first BT

Figure 2. Demonstration of CPC markers in axial images (white arrows). Ureteral stents (black arrows) were inserted in patients 1 and 3 for hydronephrosis. Column 1: TPCT obtained 2 days before EBRT; column 2: CBCT obtained a day before first BT; column 3: MRI before first BT were obtained within 24 hours after the CBCT in column 2.

solid markers were mainly developed to decrease streaking artifacts on CT $(7,11)$.

The clinical use of liquid fiducial markers in radiotherapy was reported recently, and we have summarized them in
Table III (12-19). The majority of the reports were on lipiodol, an iodized poppy-seed oil contrast medium (20), used for image guidance in the boost of bladder tumor irradiation. On $2 \mathrm{D}-\mathrm{kV}$ images of the bladder, lateral 
Table III. Clinical use of injectable markers in published papers.

\begin{tabular}{|c|c|c|c|c|c|c|c|}
\hline $\begin{array}{l}\text { Author (year), } \\
\text { reference }\end{array}$ & Marker & $\begin{array}{c}\text { Clinical } \\
\text { application }\end{array}$ & $\begin{array}{c}\text { Number of } \\
\text { patients }\end{array}$ & $\begin{array}{l}\text { Single injected } \\
\text { volume }(\mathrm{ml})\end{array}$ & $\begin{array}{c}\text { Number of } \\
\text { injections }\end{array}$ & Gauge & $\begin{array}{l}\text { Modality of imaging } \\
\text { for IGRT study }\end{array}$ \\
\hline Dudouet (2001) (14) & Lipiodol & Prostate cancer & 37 & $1.0-2.0$ & 4 & 22 & $2 \mathrm{D}-\mathrm{kV}$ \\
\hline Pos (2009) (17) & Lipiodol & Bladder cancer & 40 & 0.25 & $3-5$ & 15 & $\mathrm{CBCT}$ \\
\hline Chai (2010) (13) & Lipiodol & Bladder cancer & 15 & 0.25 & $2-4$ & - & CBCT \\
\hline Sondergaard (2010) (19) & Lipiodol & Bladder cancer & 5 & $0.25-0.5$ & $4-6$ & - & CBCT, 2D-kV \\
\hline Meijer (2012) (16) & Lipiodol & Bladder cancer & 20 & - & $3-5$ & 23 & СBCT \\
\hline Freilich (2014) (15) & Lipiodol & Bladder cancer & 5 & 0.5 & $20-30$ & 23 & $2 \mathrm{D}-\mathrm{kV}$ \\
\hline Rose (2014) (18) & Lipiodol & Lung tumor & 4 & $0.5-1.0$ & - & 25 & CBCT \\
\hline Bair (2015) (12) & Hydrogel & Gynecologic cancers & 19 & $0.1-0.4$ & 4 & 18 & $\begin{array}{l}\text { CT based BT, } \\
\text { MRI based BT }\end{array}$ \\
\hline Present report & $\mathrm{CPC}$ & Cervical cancer & 6 & $0.1-0.2$ & $3-5$ & 19 & CBCT, MRI \\
\hline
\end{tabular}

CPC: Calcium phosphate cement; CBCT: cone beam CT; BT: brachytherapy; 2D-kV: 2-dimensional kilovoltage X-ray.

projections of the bony structures of the pelvis made visualization of lipiodol very difficult, and only the anteroposterior projections showed lipiodol clearly with 0.25 $0.5 \mathrm{ml}$ injections (19). To be visible in the lateral projections on $2 \mathrm{D}-\mathrm{kV}$ images, $0.5-2 \mathrm{ml}$ of lipiodol was injected $(14,15)$. However, to decrease the artifacts on the TPCT and CBCT scans, it was necessary to limit the volume of the marker (19, 21). In addition the large artifacts on the CT images adversely affect the treatment planning system's ability to calculate accurate dose distributions (21). Bair et al. reported the first clinical use of a hydrogel marker, polyethylene glycol hydrogel micro particles containing covalently bound iodine. This marker was used to assist with 3D-based BT in gynecologic malignancies by visualizing the target. The hydrogel material was injected at volumes ranging from 0.1 to $0.4 \mathrm{ml}$, and they concluded that injecting $\geq 0.4 \mathrm{ml}$ of the hydrogel into the desired anatomic location is necessary to achieve unequivocal visibility on CT or MRI (12). In this study, we injected 0.1 to $0.2 \mathrm{ml}$ which were the smallest injection volumes compared with the other studies and we did not evaluate $2 \mathrm{D}-\mathrm{kV}$ images. Further investigation is necessary to visualize the marker in $2 \mathrm{D}-\mathrm{kV}$ and investigate whether an injection volume $>0.2 \mathrm{ml}$ could improve visibility on MRI.

Two of the greatest advantages of using a liquid marker are that implantation can be introduced with thinner needles than solid fiducial markers and the marker size can be customized by the injection volume (21). As shown in Table III, 15-25-gauge needles were used for the lipiodol markers and 18-gauge was used for the hydrogel markers. We used a 19-gauge needle which was a smaller caliber needle than the one used for GM $(12,14)$. Discomfort for patients and the risk of hemorrhage could be decreased using smaller needles.

Although absorbability and the ability to create 3D structures of liquid markers are considered to be advantages, there is a consideration that changes in the size and shape of markers during the course of radiation therapy may cause disadvantages for IGRT. Even the demarcations of lipiodol markers were clearly visible on CT and CBCT, they were slowly absorbed over time during the course of RT $(17,19)$. Chai et al. stated that diffusion or deformation of a single large lipiodol spot could lead to a bias (13). Although it is difficult to conduct evaluation with a limited slice thickness and voxel dimension, we could identify each CPC marker by the size and shape on CBCT during EBRT.

Zeng et al. reported that $46(35 \%)$ of 131 prostate patients had clearly defined calcifications in the prostate that were unambiguous even on low-dose CBCT (22). These patients had calcifications $>2 \mathrm{~mm}$ in each direction either inside the prostate, or near the prostate border. The stability of the calcifications was investigated using the geometric center of three GMs as a reference, which is considered a surrogate of the prostate mass center. They concluded that calcifications might be a reliable natural landmark to replace seeds to localize the prostate during CBCT-guided treatment and to avoid artifacts on CT images with increasing accuracy in target delineation and dose calculation. Calcifications in the prostate were also used to evaluate the soft-tissue automatic gray-value match (23) and different similarity metrics to determine the most accurate prostate and seminal vesicle localization on daily CBCT (24).

CPC is used to fill bone voids in dental, orthopedic and craniofacial applications. Several formulations of CPC have been approved worldwide for clinical use (5). Biopex-R consists of powdered and liquid components. The powder contains $75 \%$ alphatricalcium phosphate, $18 \%$ tetracalcium phosphate, $5 \%$ dicalcium phosphate dihydrate, and $2 \%$ hydroxyapatite (HA), and the liquid contains $12 \%$ sodium succinate, $5 \%$ sodium chondroitin sulfate, and $83 \%$ distilled water. The injectable CPC paste is prepared by manual mixing of the two components for $1 \mathrm{~min}$. This paste is self- 
hardening within $10 \mathrm{~min}$ and recrystallizes to $\mathrm{HA}$ in the living body without an organic content. They show very little bioresorbability in the living body because their final reaction product is mainly HA (4). HA is the primary mineral component of teeth and bone, being safe in the living body (3), and its enables the avoidance of artifacts on CT images. Shiotani et al. reported the implantation of CPC into soft tissue as injection laryngoplasty and revealed that the CPC was safe, non-absorbable, and effective (25).

The use of MRI has become important in treatment planning for both gynecologic and prostate cancer. Macroscopic tumor extension was visualized on MRI with a high signal intensity mass on fast or turbo spin echo sequence T2 in gynecologic cancer, and was limited when using only CT (26). T2-weighted MRI was also used as the gold standard in treatment planning for prostate cancer, because of overestimation of prostate volume with CT images compared with MRI and tumor localization was visualized on MRI (27, 28). Parker et al. investigated the most suitable gold alloy marker composition for CT-MR image fusion purposes in prostate cancer. The marker localization was limited by the poor definition of the markers on MRI, and the use of fiducial markers for co-registration purposes raises the possibility of marker-induced distortion of MRI (27). Nyholm et al. suggested that internal markers appear clearly on gradient echo sequences, while there is more difficulty to identify them on T2-weighted fast or turbo spin echo sequences. The visibility of the markers was increased when the TE time was reduced, yielding a higher signal but compromising the T2weighted contrast (29). MRI is dependent on the presence of hydrogen, but the hydrogen contents of CPC markers is very low. CPC markers are shown as black or very dark because of their low signal intensity, which is similar to that of cortical bone. Prostatic calculi are composed of calcium phosphate and organic content (30), and are usually not clearly visible on MRI. In our study, 13 CPC markers without organic content were visible as black, and 3 CPC markers adjacent to the endocervical canal were not visible, probably because of the presence of organic content.

Fiducial markers have been used in gynecological radiation therapy $(11,12,31)$. Marked marker loss was reported by Kaatee et al. Although markers were attached to healthy parts of the cervix and attachment on the tumor was avoided, almost half of their markers were lost: half before the start of EBRT and half during EBRT. They concluded that the reason for the observed loss of markers might be tumor growth or regression, especially because it was not always possible to attach all markers to healthy cervix tissue (31). Although we did not inject CPC markers into normal tissue and placements of applicators for BT sources were performed in addition to EBRT, our marker loss was less marked compared with the results found by them. All markers were lost before or during EBRT in one patient with the lowest hemoglobin level. The reason for the marker loss might be bleeding from carcinoma of the uterine cervix.

To ensure at least a $95 \%$ dose to (on average) $99 \%$ of the CTV, Stroom et al. determined that $2 \Sigma+0.7 \sigma$ is required for a margin between the CTV and PTV (6). Our CTV-toPTV margins for the cervix calculated by their formula were $1.8,13.1$, and $6.1 \mathrm{~mm}$ in the left-right, dorsal-ventral, and cranial-caudal directions, respectively. Our results were lower for the left-right and cranial-caudal directions than in the report by Langerak et al. $(7.1,11.9$, and $11.5 \mathrm{~mm}$, respectively) and Kaatee et al. (8.9, 9.7, and $10.8 \mathrm{~mm}$, respectively) $(11,31)$. Chan et al. reported that inter-scan motion was the greatest at the fundus of the uterus, lower along the canal, and the lowest at the cervical os using serial cinematic MRI scans (32). Probably our markers were injected more central in the tumor near the cervical os, whereas others reported that markers were implanted in normal tissue away from the center of the cervical tumor.

This is the first report of non-metallic fiducial markers by injecting CPC paste into tumors of the cervix. They are visualized on CBCT and MRI while reducing marker loss and artifacts. Future studies using CPC markers for IGRT are warranted for cervical cancer and other clinical manifestations.

\section{Conflicts of Interest}

The Authors declare that there is no conflict of interest in regard to this study.

\section{Acknowledgements}

This work was supported by Japan Society for the Promotion of Science Grants-in-Aid for Scientific Research (25461922, and 17K10487).

\section{References}

1 Moseley DJ, White EA, Wiltshire KL, Rosewall T, Sharpe MB, Siewerdsen JH, Bissonnette JP, Gospodarowicz M, Warde P, Catton $\mathrm{CN}$ and Jaffray DA: Comparison of localization performance with implanted fiducial markers and cone-beam computed tomography for on-line image-guided radiotherapy of the prostate. Int J Radiat Oncol Biol Phys 67: 942-953, 2007.

2 Soete G, De Cock M, Verellen D, Michielsen D, Keuppens F and Storme G: X-ray-assisted positioning of patients treated by conformal arc radiotherapy for prostate cancer: comparison of setup accuracy using implanted markers versus bony structures. Int J Radiat Oncol Biol Phys 67: 823-827, 2007.

3 Costantino PD, Friedman CD, Jones K, Chow LC and Sisson GA: Experimental hydroxyapatite cement cranioplasty. Plast Reconstr Surg 90: 174-185, 1992.

4 Musha Y, Umeda T, Yoshizawa S, Shigemitsu T, Mizutani K and Itatani $\mathrm{K}$ : Effects of blood on bone cement made of calcium phosphate: problems and advantages. J Biomed Mater Res B Appl Biomater 92: 95-101, 2010. 
5 Ambard AJ and Mueninghoff L: Calcium phosphate cement: review of mechanical and biological properties. J Prosthodont 15: 321-328, 2006.

6 Stroom JC, de Boer HC, Huizenga H and Visser AG: Inclusion of geometrical uncertainties in radiotherapy treatment planning by means of coverage probability. Int J Radiat Oncol Biol Phys 43: 905-919, 1999.

7 Habermehl D, Henkner K, Ecker S, Jakel O, Debus J and Combs SE: Evaluation of different fiducial markers for image-guided radiotherapy and particle therapy. J Radiat Res 54: i61-68, 2013.

8 Owen R, Kron T, Foroudi F, Cox J, Zhu L, Cramb J, Sparks L and Duchesne G: The detectability and localization accuracy of implanted fiducial markers determined on in-room computerized tomography (CT) and electronic portal images (EPI). Med Dosim 33: 226-233, 2008.

9 Chow JC and Grigorov GN: Dose measurements near a nonradioactive gold seed using radiographic film. Phys Med Biol 50: N227-234, 2005.

10 Chow JC and Grigorov GN: Monte Carlo simulations of dose near a nonradioactive gold seed. Med Phys 33: 4614-4621, 2006.

11 Langerak T, Mens JW, Quint S, Bondar L, Heijkoop S, Heijmen $\mathrm{B}$ and Hoogeman M: Cervix motion in 50 cervical cancer patients assessed by daily cone beam computed tomographic imaging of a new type of marker. Int J Radiat Oncol Biol Phys 93: 532-539, 2015.

12 Bair RJ, Bair E and Viswanathan AN: A radiopaque polymer hydrogel used as a fiducial marker in gynecologic-cancer patients receiving brachytherapy. Brachytherapy 14: 876-880, 2015.

13 Chai X, van Herk M, van de Kamer JB, Remeijer P, Bex A, Betgen A, De Reijke TM, Hulshof MC, Pos FJ and Bel A: Behavior of lipiodol markers during image guided radiotherapy of bladder cancer. Int J Radiat Oncol Biol Phys 77: 309-314, 2010.

14 Dudouet P, Portalez D, Lhez JM, Elman B, Larroque JM, Bachaud JM, Redon A and Ribot JF: Trans-rectal ultrasonography (TRUS) with lipiodol injection for localization of the prostatic apex before radiotherapy planning. Radiother Oncol 61: 135-141, 2001.

15 Freilich JM, Spiess PE, Biagioli MC, Fernandez DC, Shi EJ, Hunt DC, Gupta S and Wilder RB: Lipiodol as a fiducial marker for image-guided radiation therapy for bladder cancer. Int Braz J Urol 40: 190-197, 2014.

16 Meijer GJ, van der Toorn PP, Bal M, Schuring D, Weterings J and de Wildt M: High precision bladder cancer irradiation by integrating a library planning procedure of 6 prospectively generated SIB IMRT plans with image guidance using lipiodol markers. Radiother Oncol 105: 174-179, 2012.

17 Pos F, Bex A, Dees-Ribbers HM, Betgen A, van Herk M and Remeijer P: Lipiodol injection for target volume delineation and image guidance during radiotherapy for bladder cancer. Radiother Oncol 93: 364-367, 2009.

18 Rose M, Siva S, Ball D, Irving LB and Steinfort DP: Bronchoscopic delivery of lipiodol as a fiducial marker in lung tumors before radiotherapy. J Thorac Oncol 9: 1579-1583, 2014.

19 Sondergaard J, Olsen KO, Muren LP, Elstrom UV, Grau C and Hoyer M: A study of image-guided radiotherapy of bladder cancer based on lipiodol injection in the bladder wall. Acta Oncol 49: 1109-1115, 2010.

20 Bonnemain B and Guerbet M: [The history of Lipiodol (19011994) or How a medication may evolve with the times]. Rev Hist Pharm (Paris) 42: 159-170, 1995.
21 Scherman Rydhog J, Irming Jolck R, Andresen TL and Munck Af Rosenschold P: Quantification and comparison of visibility and image artifacts of a new liquid fiducial marker in a lung phantom for image-guided radiation therapy. Med Phys 42: 2818-2826, 2015.

22 Zeng GG, McGowan TS, Larsen TM, Bruce LM, Moran NK, Tsao JR and MacPherson MS: Calcifications are potential surrogates for prostate localization in image-guided radiotherapy. Int J Radiat Oncol Biol Phys 72: 963-966, 2008.

23 Smitsmans MH, de Bois J, Sonke JJ, Betgen A, Zijp LJ, Jaffray DA, Lebesque JV and van Herk M: Automatic prostate localization on cone-beam CT scans for high precision image-guided radiotherapy. Int J Radiat Oncol Biol Phys 63: 975-984, 2005.

24 Kim J, Hammoud R, Pradhan D, Zhong H, Jin RY, Movsas B and Chetty IJ: Prostate localization on daily cone-beam computed tomography images: accuracy assessment of similarity metrics. Int J Radiat Oncol Biol Phys 77: 1257-1265, 2010.

25 Shiotani A, Okubo K, Saito K, Fujimine T, Tomifuji M, Ikeda A, Araki K and Momoshima S: Injection laryngoplasty with calcium phosphate cement. Otolaryngol Head Neck Surg 140: 816-821, 2009

26 Haie-Meder C, Potter R, Van Limbergen E, Briot E, De Brabandere M, Dimopoulos J, Dumas I, Hellebust TP, Kirisits C, Lang S, Muschitz S, Nevinson J, Nulens A, Petrow P, WachterGerstner N and Gynaecological GECEWG: Recommendations from Gynaecological (GYN) GEC-ESTRO Working Group (I): concepts and terms in 3D image based 3D treatment planning in cervix cancer brachytherapy with emphasis on MRI assessment of GTV and CTV. Radiother Oncol 74: 235-245, 2005.

27 Parker CC, Damyanovich A, Haycocks T, Haider M, Bayley A and Catton $\mathrm{CN}$ : Magnetic resonance imaging in the radiation treatment planning of localized prostate cancer using intraprostatic fiducial markers for computed tomography coregistration. Radiother Oncol 66: 217-224, 2003.

28 Hasumi M, Suzuki K, Taketomi A, Matsui H, Yamamoto T, Ito $\mathrm{K}$, Kurokawa K, Aoki J, Endo $\mathrm{K}$ and Yamanaka H: The combination of multi-voxel MR spectroscopy with MR imaging improve the diagnostic accuracy for localization of prostate cancer. Anticancer Res 23: 4223-4227, 2003.

29 Nyholm T, Nyberg M, Karlsson MG and Karlsson M: Systematisation of spatial uncertainties for comparison between a MR and a CT-based radiotherapy workflow for prostate treatments. Radiat Oncol 4: 54, 2009.

30 Torres Ramirez C, Aguilar Ruiz J, Zuluaga Gomez A, Espuela Orgaz R and Del Rio Samper S: A crystallographic study of prostatic calculi. J Urol 124: 840-843, 1980.

31 Kaatee RS, Olofsen MJ, Verstraate MB, Quint S and Heijmen $\mathrm{BJ}$ : Detection of organ movement in cervix cancer patients using a fluoroscopic electronic portal imaging device and radiopaque markers. Int J Radiat Oncol Biol Phys 54: 576-583, 2002.

32 Chan P, Dinniwell R, Haider MA, Cho YB, Jaffray D, Lockwood G, Levin W, Manchul L, Fyles A and Milosevic M: Inter- and intrafractional tumor and organ movement in patients with cervical cancer undergoing radiotherapy: a cinematic-MRI point-of-interest study. Int J Radiat Oncol Biol Phys 70: 1507-1515, 2008.

Received June 30, 2018

Revised July 14, 2018

Accepted July 16, 2018 